\title{
ESTRATEGIA DIDÁCTICA PARA RESOLVER PROBLEMAS EN LAS CLASES DE MATEMÁTICA
}

\author{
AUTORES: Raquel Vera Velázquez ${ }^{1}$ \\ Marcos Manuel Manobanda Guamán ${ }^{2}$ \\ Kirenia Maldonado Zúñiga ${ }^{3}$ \\ Yoiler Batista Garcet4 \\ Alfredo Lesvel Castro Landin ${ }^{5}$
}

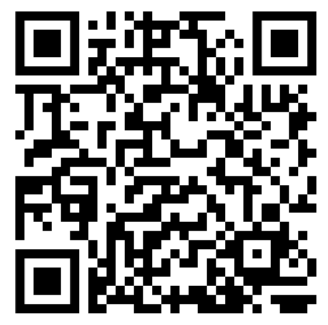

\section{DIRECCIÓN PARA CORRESPONDENCIA: (raquelita2015vera@gmail.com)}

Fecha de recepción: 15/02/2019

Fecha de aceptación: 23/04/2019

\section{RESUMEN}

Esta investigación estuvo dirigida hacia la resolución de problemas matemáticos aspecto que ha presentado dificultades en el desarrollo del proceso docente educativo, se diseñó una estrategia a partir de criterios para la enseñanza y la evaluación de los aprendizajes, derivados de teorías cognitivas, se desarrollaron tópicos relativos al tema de ecuaciones lineales que conducen a la resolución de problemas en la asignatura de álgebra en él primer semestre de Ingeniería Agropecuaria. En este trabajo se analizan, en forma descriptiva, los resultados de una encuesta aplicada a una muestra aleatoria representativa de los 96 estudiantes que participaron en la experiencia con el objetivo de determinar en qué medida se cumplieron en la práctica los criterios establecidos en una estrategia didáctica aplicada en las clases de Matemática. La misma enfatiza la participación activa del estudiante durante el uso en las clases de un material elaborado sobre resolución de problemas aplicados al contenido: ecuaciones y funciones. Al momento de diseñar la estrategia se reconoció que era necesario tomar decisiones respecto a actividades de la práctica docente que corresponden a la preparación de las clases (antes) y (durante) la clase. Se puede apreciar que la importancia relativa de las distintas dimensiones osciló entre $65 \%$ y 83\%. Los valores más altos estarían indicando que los estudiantes: aprueban el material didáctico propuesto, mostrándose complacidos por tener una participación activa en la clase y por el trabajo grupal, están de acuerdo con el rol desempeñado por el docente como guía del aprendizaje y como trasmisor del saber.

\section{DIDACTIC STRATEGY FOR RESOLVING PROBLEMS USED IN MATHEMATICS CLASSES}

\footnotetext{
1 Máster en Ciencias de la Educación. Universidad de Ciencias Pedagógicas, Las Tunas Cuba.raquelita2015vera@gmail.com 2 Máster en Docencia Universitaria. Universidad Laica. Eloy Alfaro de Manabì.marcos.manobanda@unesum.edu.ec 3 Máster en Ciencias de la Educación.Universidad de Ciencias Pedagógicas, Holguín Cuba.kmzmaldonado@gmail.com 4 Máster en Ciencias de la Educación.Universidad de Ciencias Pedagògicas,Las Tunas yoiler.batista@unesum.edu.ec 5 Máster en Ciencias Agrícolas. Universidad Camilo Cienfuegos, Matanzas.alfredolandin.55@gmail.com
} 
Raquel Vera Velázquez1, Marcos Manuel Manobanda Guamán2, Kirenia Maldonado Zúñiga3, Yoiler Batista Garcet4,Alfredo Lesvel Castro Landin 5

PALABRAS CLAVE: Problemas, matemáticos, ecuaciones, funciones y didáctica.

\section{ABSTRACT}

This research was aimed at solving mathematical problems, an aspect that has presented difficulties in the development of the educational process, a strategy was designed based on criteria for teaching and the evaluation of learning, derived from cognitive theories, relative topics were developed to the topic of linear equations that lead to the resolution of problems in the subject of algebra in the first semester of Agricultural Engineering. In this paper, the results of a survey applied to a representative random sample of the 96 students who participated in the experience are analyzed in order to determine to what extent the criteria established in a didactic strategy were met in practice. Applied in Mathematics classes. It emphasizes the active participation of the student during the use in the classes of a material developed on problem solving applied to the content: equations and functions. At the time of designing the strategy it was recognized that it was necessary to make decisions regarding activities of the teaching practice that correspond to the preparation of the classes (before) and (during) the class. It can be seen that the relative importance of the different dimensions ranged between $65 \%$ and $83 \%$. The highest values would be indicating that the students: approve the proposed teaching material, being pleased to have an active participation in the class and for the group work, they are in agreement with the role played by the teacher as a guide of learning and as a transmitter of the to know.

KEYWORDS: Problems, mathematics, equations, functions and didactics

\section{INTRODUCCIÓN}

Álgebra es una de las asignaturas del primer semestre de las carreras de Ingeniería en la facultada de ciencias naturales y de la agricultura que se cursan en la Universidad Estatal del Sur de Manabí. En ella se desarrollan los conceptos básicos y algoritmos de trabajo del trabajo con variable y se aplica a la resolución de problemas en el entorno agropecuario, forestal y medioambiental.

En los diagnósticos aplicados a los estudiantes de la carrera agropecuaria se pudieron identificar algunas de las características de los procesos de enseñanza y aprendizaje del álgebra, correspondientes a períodos lectivos anteriores donde: las clases teóricas eran del tipo magistral dialogada; el excesivo número de estudiantes determinaba que el docente fuera el protagonista principal y la actividad del estudiante fuera mínima (González de Galindo, 2003). Estas clases respondían a la forma dominante de instrucción que Gregg (1995) denomina "instrucción centrada en el profesor", en la que es el docente quien habla principalmente y determina cómo se usa el tiempo en la clase, el trabajo con todo el grupo prevalece sobre el trabajo individual o en grupos pequeños y los estudiantes se sientan en filas frente al profesor.

La evaluación de los aprendizajes se realizaba por exigencias de la gestión académica y no por una necesidad pedagógica (González de Galindo, 2003; Villalonga de García, 2003). La función de control estaba sobredimensionada, subordinando a las demás funciones pedagógicas de la evaluación: directiva del proceso, predictiva, reguladora de la actividad del estudiante y formativa (González Pérez, 2000); la comunicación entre los distintos agentes del proceso educativo evidenciaba falencias,

96 UNESUM-Ciencias. Publicación cuatrimestral. Vol. 3, Año 2018, No. 2 (agosto) 
ya que la educación no se interpretaba como un proceso de interacción y comunicación (Rodrigo y Arnay, 1997; González de Galindo et al, 2006);

La clase, respondía a la idea de un plan, que conducía el proceso de enseñanza aprendizaje. Los contenidos teóricos y la ejercitación correspondiente, desarrollaban en los estudiantes habilidades de cálculo y de resolución de ejercicios formales. No resultaba suficiente la cantidad de problemas que se proponían a los estudiantes para incentivar el razonamiento Durante el semestre de la asignatura Álgebra, los estudiantes evidenciaban fallas en la solidez de los conocimientos básicos adquiridos en la Básica y el Bachillerato. La problemática detectada, dio origen a la estrategia sobre "Metodología de la Enseñanza y Evaluación que favorecen aprendizajes significativos” previo (González de Galindo, Marcilla y Villalonga de García, 2006). Se comenzó por elaborar un marco teórico de referencia siguiendo principios de teorías cognitivas del aprendizaje, enunciados por Piaget, Ausubel, Vigotsky, entre otros y la aplicación del modelo educativo de la Universidad Estatal del Sur de Manabí y el rediseño de la carrera de Ingeniería agropecuaria.

Atendiendo al objetivo de este trabajo se consideró oportuno apuntar hacia la resolución de problemas matemáticos aspecto que ha presentado dificultades en el desarrollo del proceso docente educativo, se diseñó una estrategia a partir de criterios para la enseñanza y la evaluación de los aprendizajes, derivados de teorías cognitivas, se implementó en el primer semestre de 2018, en el material empleado, se desarrollaron tópicos relativos al tema de ecuaciones lineales que conducen a la resolución de problemas en la asignatura de álgebra.

\section{DESARROLLO}

\section{MATERIALES Y MÉTODOS}

En este trabajo se analizan, en forma descriptiva, los resultados de una encuesta aplicada a una muestra aleatoria representativa de los 96 de primer semestre de ingeniería agropecuaria, estudiantes que participaron en la experiencia con el objetivo de determinar en qué medidas se cumplieron en la práctica los criterios establecidos en una estrategia didáctica aplicada en las clases de Matemática.

\section{ESTRATEGIA DIDÀCTICA}

Las Tendencias Pedagógicas seleccionadas para fundamentar teóricamente la estrategia didáctica fueron el Enfoque Histórico Cultural de Vigotsky con la Teoría de la Actividad de Leontiev y el Enfoque Cognitivo con las Teorías Psicogenética de Piaget y del Aprendizaje significativo de Ausubel (Coll, Palacios y Marchesi, 1992; Moreira, 1997; Pérez Gómez, 1992). El estudio de estas teorías permitieron elaborar el marco teórico de referencia. Se adoptó como modelo de aprendizaje, el que sostienen las teorías cognitivas, las que consideran que el origen de los cambios es interno y atribuyen importancia relevante tanto al significado de los aprendizajes como a la influencia de los factores sociales. Las premisas teóricas de cada una de las teorías seleccionadas fueron:

Teoría Psicogenética de Piaget (constructivismo) como modelo utilizado en nuestra universidad: considera que el conocimiento se construye a través de la interacción entre el sujeto y el medio. En la construcción del conocimiento asume que:

a) La fuente del mismo es la actividad significativa del sujeto. 
b) El proceso de construcción depende del nivel cognitivo inicial y de las estructuras mentales del individuo y se desarrolla a partir de la actividad, reflexión y confrontación social.

c) El acceso al conocimiento no es cerrado.

d) El docente es el sujeto facilitador del aprendizaje constructivo.

e) En el desarrollo de las estructuras cognitivas es necesario conceder importancia al error producido en la adquisición del conocimiento y a las experiencias sociales.

f) El aprendizaje depende del tipo de actividades realizadas.

g) Los conflictos desempeñan un papel importante en el aprendizaje (Ortiz Hurtado, 1999).

Enfoque histórico cultural de Vigotsky: La tesis central de esta teoría es la ley genética fundamental del desarrollo: “Toda función psíquica aparece en acción dos veces, primero en el plano social (plano interpsicológico) y posteriormente en el individual (plano intrapsicológico)" (Hernández Fernández, 2001: 36). Esta ley valida lo siguiente: 1) se reconoce el origen social del proceso de aprendizaje.

2) existe una dinámica entre la actividad externa y la interna.

3) el aprendizaje tiene una estructura mediatizada. Las características más relevantes de esta teoría son: el aprendizaje está en función de la comunicación y el desarrollo; el desarrollo sigue al aprendizaje y está influido por los aprendizajes guiados a través de la enseñanza sistemática; es relevante la apropiación del bagaje cultural en la formación de las estructuras formales; el motor fundamental del desarrollo es la actividad del individuo.

Teoría del Aprendizaje Significativo de Ausubel: Esta teoría centra su atención en la naturaleza significativa del aprendizaje humano escolarizado, investigando los procesos cognitivos internos que conducen a él. Los conceptos básicos de la misma son: estructura cognoscitiva (sistema de conceptos y proposiciones organizados jerárquicamente), conocimientos previos y significado lógico (la estructura interna del material de aprendizaje debe estar ordenada jerárquicamente) y psicológico (el material potencialmente significativo se convierte en un contenido nuevo de aprendizaje, al asimilarse y relacionarse con los conceptos pertinentes de la estructura cognoscitiva del sujeto). Ausubel afirma que el aprendizaje significativo requiere de un material potencialmente significativo (significado lógico) y de una actitud favorable para ese aprendizaje (significado psicológico).

A partir de las premisas del marco teórico enunciado se derivaron los criterios que debieran guiar el proceso de enseñanza, la selección y forma de presentación de las distintas actividades que se propongan al estudiante, la modalidad del desarrollo de las clases, el diseño de los instrumentos de evaluación y el proceso de evaluación del aprendizaje de la Matemática . Estos criterios son (Villalonga de García, González de Galindo, 2005): Durante el proceso de enseñanza y aprendizaje el docente debería:

1-Favorecer el protagonismo activo del estudiante como responsable de su aprendizaje.

2- Propiciar el intercambio grupal de significados, intentando satisfacer las pautas relativas a comunicación establecidas en los Principios y Estándares para la Educación Matemática. (Ministerio Educación, 2016).

98 UNESUM-Ciencias. Publicación cuatrimestral. Vol. 3, Año 2018, No. 2 (agosto) 
3-Otorgar mayor dinamismo al proceso de enseñanza y aprendizaje, con un ritmo que mantenga la atención y el interés, considerando a la evaluación formativa como medio para introducir los cambios pertinentes para ajustar dicho proceso a las características y capacidades de los estudiantes.

4-Presentar los contenidos de modo que facilite el desarrollo de las habilidades y destrezas propias del conocimiento matemático.

5-Favorecer el cambio del rol docente, desde el de transmisor de conocimientos ciertos y acabados, al de facilitador de aprendizajes centrados en cuestionamientos, reflexión crítica y construcción de significados, con la capacidad de generar en la clase una atmósfera de coparticipación distendida. Además, es el responsable del proceso de institucionalización, proceso en el que el maestro establece las relaciones entre los conocimientos fuertemente contextualizados construidos por el estudiante y los saberes institucionales (Moscoso Canabal, 2005).

6-Despertar el interés por los temas del Cálculo, basándose en el uso y necesidad práctica de los mismos para resolver problemas vinculados a la carrera y a la vida diaria.

7-Diseñar las actividades de clase y los instrumentos de evaluación de los aprendizajes siguiendo las pautas relativas a conexiones entre contenidos establecidas en (Ministerio Educación, (2016) y en los que se aprecie la importancia que se conceden a los aprendizajes significativos.

8-Con referencia al Criterio C2, el Estándar relativo a Comunicación establece las siguientes pautas: "Los programas de enseñanza de todas las etapas deberían capacitar a todos los estudiantes para:

- Organizar y consolidar su pensamiento matemático a través de la comunicación.

- Los procesos que deben desarrollarse en el aula para lograr el conocimiento son los siguientes: Concientización de las ideas previas y del grado de dominio de los prerrequisitos de aprendizaje.

- Confrontación de las propias ideas y de las consensuadas en el pequeño grupo.

- Introducción formal de los conceptos y enunciados de teoremas.

- Aplicación de los nuevos conceptos y enunciados de teoremas.

\section{METOODOLOGİA}

En un trabajo previo (González de Galindo, Marcilla y Villalonga de García, 2006) se describe la estrategia didáctica, la que fue diseñada considerando los criterios enunciados. La misma enfatiza la participación activa del estudiante a partir del uso durante las clases de un material instrucciones elaborado sobre resolución de problemas aplicados al contenido: ecuaciones y funciones. Al momento de diseñar la estrategia se reconoció que era necesario tomar decisiones respecto a actividades de la práctica docente que corresponden a la preparación de las clases (antes) y a la clase misma (durante). Es decir, fue necesario abocarse a dos tipos de actividades: las que forman parte de la planificación, como diseñar, elegir o modificar los problemas que se iban a plantear a los estudiantes, organizar el contenido y determinar las actividades de evaluación y las que forman parte de la gestión del proceso de enseñanza aprendizaje, como las relativas al conocimiento matemático involucrado en los problemas seleccionados y a la interacción entre el docente y los estudiantes y los estudiantes entre sí. Con respecto al (antes), se decidió incluir en el material didáctico actividades que posibilitaran a los 
Raquel Vera Velázquez1, Marcos Manuel Manobanda Guamán2, Kirenia Maldonado Zúñiga3, Yoiler Batista Garcet4,Alfredo Lesvel Castro Landin 5

estudiantes intuir, descubrir, construir y comprender los conceptos matemáticos involucrados en las mismas, con la mínima intervención del profesor. Con respecto al diseño de las actividades que forman parte de la gestión del proceso de enseñanza aprendizaje (durante), se decidió que la estrategia se basara en el modelo de trabajo en el aula que pone énfasis en la naturaleza individual y colectiva del proceso de aprendizaje. Por ello, se alternaron espacios de trabajo individual con otros destinados a la interacción grupal. Se planificaron seis espacios para el trabajo en el aula:

Fase 1: Indicaciones del docente y análisis de la guía de práctica.

Fase 2: Reflexión personal.

Fase 3: Discusión entre grupo.

Fase 4: Evaluación grupal.

Fase 5: Institucionalización del saber.

Fase 6: Resolución de situaciones problemáticas.

De acuerdo a los momentos establecidos es posible apreciar que los procesos que deben desarrollarse en el aula para lograr el conocimiento son los siguientes:

- Concientización de las ideas previas y del grado de dominio de los prerrequisitos de aprendizaje.

- Confrontación de las propias ideas y de cada grupo.

- Introducción formal de los conceptos y enunciados de teoremas.

- Aplicación de los nuevos conceptos y enunciados de teoremas.

3.1 Implementación de la estrategia didáctica

Se llevó a cabo en el primer semestre de 2018, después de haber desarrollado durante las primeras tres semanas contenidos relativos a los tópicos de ecuaciones y funciones. Los estudiantes que participaron fueron 96 del primer semestre de ingeniería agropecuaria; se les comunicó que participarían de una experiencia didáctica para abordar el tema ecuaciones y funciones. Fueron distribuidos para las clases teóricas en tres grupos de aproximadamente 32 estudiantes cada uno y para las clases prácticas en 8 equipos de 4 estudiantes en cada grupo.

Evaluación de la estrategia didáctica

Conocer los puntos de vista de la experiencia implementada en el primer semestre de 2018, permitiría inferir si la misma se había ajustado a los lineamientos propios de un aprendizaje significativo. Para evaluar la experiencia se consideró importante examinar tanto el rendimiento académico de los estudiantes en los contenidos desarrollados en la guía, como cuestiones relativas al proceso educativo: desarrollo de las actividades, relaciones que se establecieron entre los implicados, vivencias de los estudiantes, etc.

La recolección de los datos se hizo recurriendo a distintas fuentes y procedimientos. Los instrumentos empleados fueron:

a) Instrumentos diseñados para evaluar los aprendizajes de los estudiantes:

- Examen correspondiente al 1er parcial de Álgebra (Julio 2018)

- Examen segundo parcial final (septiembre 2018).

100 UNESUM-Ciencias. Publicación cuatrimestral. Vol. 3, Año 2018, No. 2 (agosto) 
Es posible analizar que se apreciarían mejoras en el rendimiento académico logrado por los estudiantes que participaron de la experiencia, comparado con el alcanzado por los estudiantes del semestre (noviembre 2017- marzo 2018), (previa a esta comparación, se comprobó la homogeneidad de ambas poblaciones de estudiantes con respecto a ciertas variables consideradas relevantes: sexo, edad, institución educativa de procedencia, zona de origen, condición de recursante y rendimiento académico en los examenes aplicados .

b) Instrumentos destinados a indagar sobre la práctica didáctica en el aula:

-Control y seguimiento de las evaluaciones sistemáticas de los estudiantes (González de Galindo y Villalonga de García, 2007).

\section{ANÀLISIS DE LOS DATOS}

El análisis de los datos provenientes de la observación sistemática participante realizada por el profesor de teoría que llevó a cabo la experiencia, evidenció que existiría satisfacción con la nueva estrategia, al haberse mejorado la comunicación, la participación de los estudiantes, el desarrollo del pensamiento lógico y el interés por el contenido desarrollado, comprobándose que, en buena medida, se cumplieron los criterios orientadores de la enseñanza de la Matemática deducidos del marco teórico.

-Cuestionario a los estudiantes: este trabajo presenta el análisis de los datos provenientes de este último instrumento.

El estudio realizado es consistente con la orientación interpretativa, aun cuando se apoya en estadísticas sobre las respuestas al cuestionario.

Considerando los criterios orientadores de la enseñanza de la Matemática derivados del marco teórico se elaboró un cuestionario con trece ítems. Los mismos fueron formulados para conocer las opiniones de los estudiantes sobre los siguientes aspectos: clases teóricas (participación de los estudiantes y trabajo grupal, ritmo de la clase, razonamiento y conexiones entre contenidos), guía teórico-práctica (presentación de los contenidos, aplicación de la Matemática a las ciencias y a la vida diaria), rol del docente y adhesión a la metodología empleada. Cada una de estas variables, relativa a alguno o algunos de los criterios establecidos, fue medida a través de dos ítems de respuestas cerradas con tres alternativas de elección. Se optó por ítems de respuesta cerrada porque se consideró que resultaban más operativos, tanto para los estudiantes que los cumplimentaba como para el análisis de los datos que se obtuvieran. Se consideró que la coherencia de las respuestas a ambos ítems indicaría la sinceridad de las mismas. Los seis primeros ítems constituyeron la primera parte del cuestionario, la segunda parte y la pregunta trece (única pregunta en la que se solicitó a los estudiantes que justificaran la opción elegida) constituiría la tercera parte (ver tabla 1). Por lo tanto, las categorías que permitirían el análisis de los resultados fueron establecidas a priori. Se realizó una encuesta piloto a una muestra de quince estudiantes, para detectar la comprensión del texto, la importancia de las preguntas y la extensión del cuestionario. La versión definitiva del mismo, resultante de las modificaciones que se juzgaron pertinentes, fue administrada al finalizar la última clase de la experiencia y respondida anónimamente por los 96 estudiantes.

Según Samaja (2003) todo dato de cualquier investigación empírica posee una estructura de cuatro componentes: unidad de análisis, variables, valores e indicadores, denominada matriz de datos. Considera como indicador al procedimiento aplicado a 
Raquel Vera Velázquez1, Marcos Manuel Manobanda Guamán2, Kirenia Maldonado Zúñiga3, Yoiler Batista Garcet4,Alfredo Lesvel Castro Landin 5

cada dimensión de la variable para determinar su valor. Denomina espacio de atributos al conjunto de las variables relevantes elegidas para describir el objeto real de la investigación. En este caso, el espacio de atributos considerado quedó determinado por las variables y dimensiones que se especifican en la Tabla 1. Para cada variable y/o dimensión se consideró como unidad de análisis a las respuestas dadas a los dos ítems relacionados con ella.

Definición conceptual de las variables

Considerando el marco teórico y las características contextuales de la investigación se definieron conceptualmente las variables y dimensiones de la siguiente manera:

a) Clases teóricas: Clases en las que se implementó la estrategia didáctica y se desarrollaron los contenidos sobre el núcleo conceptual seleccionado.

1) Participación de los estudiantes y trabajo conjunto: oportunidades brindadas a los estudiantes para intervenir en la clase formulando o respondiendo preguntas del docente y para interactuar con sus compañeros a fin de completar la guía y resolver las distintas actividades.

2) Ritmo de la clase: rapidez con que el docente desarrolló los contenidos incluidos en el material didáctico.

3) Desarrollo del pensamiento lógico: exigencias de razonamiento motivadas por la estructuración de las actividades de la guía y por las preguntas formuladas por el docente.

b) Guía teórico-práctica: material didáctico elaborado según lineamientos constructivistas y que fuera empleado en las clases.

1) Presentación y desarrollo de los contenidos: forma en la que se estructuraron los contenidos conceptuales y procedimentales del tema elegido.

2) Relación de la teoría con la práctica profesional y la vida diaria: visión que tienen los estudiantes acerca de la Matemática, resultante del tipo de actividades incluida en el material instruccional y/o desarrollada por el docente en las clases.

c) Rol del docente: función desempeñada por el docente como facilitador en el proceso de apropiación del conocimiento y como institucionalizador del saber (Moscoso Canabal, 2005).

d) Afección por la nueva metodología: grado de aceptación, por parte de los estudiantes, de la estrategia didáctica implementada. La definición operacional de las variables puede apreciarse en la Tabla 1.

VARIABLES Y DIMENSIONES POR INDICADORES

\begin{tabular}{|l|l|l|}
\hline VARIABLES & DIMENSIÓN (ITEMS) & INDICADORES \\
\hline CLASES & PARTICIPACIÓN Y TRABAJO GRUPAL & Muy conforme (3): si la opción elegida como respuesta fue \\
TEÓRICAS & sí. & - Regularmente conforme (2): si la opción elegida fue más \\
\hline
\end{tabular}

102 UNESUM-Ciencias. Publicación cuatrimestral. Vol. 3, Año 2018, No. 2 (agosto) 


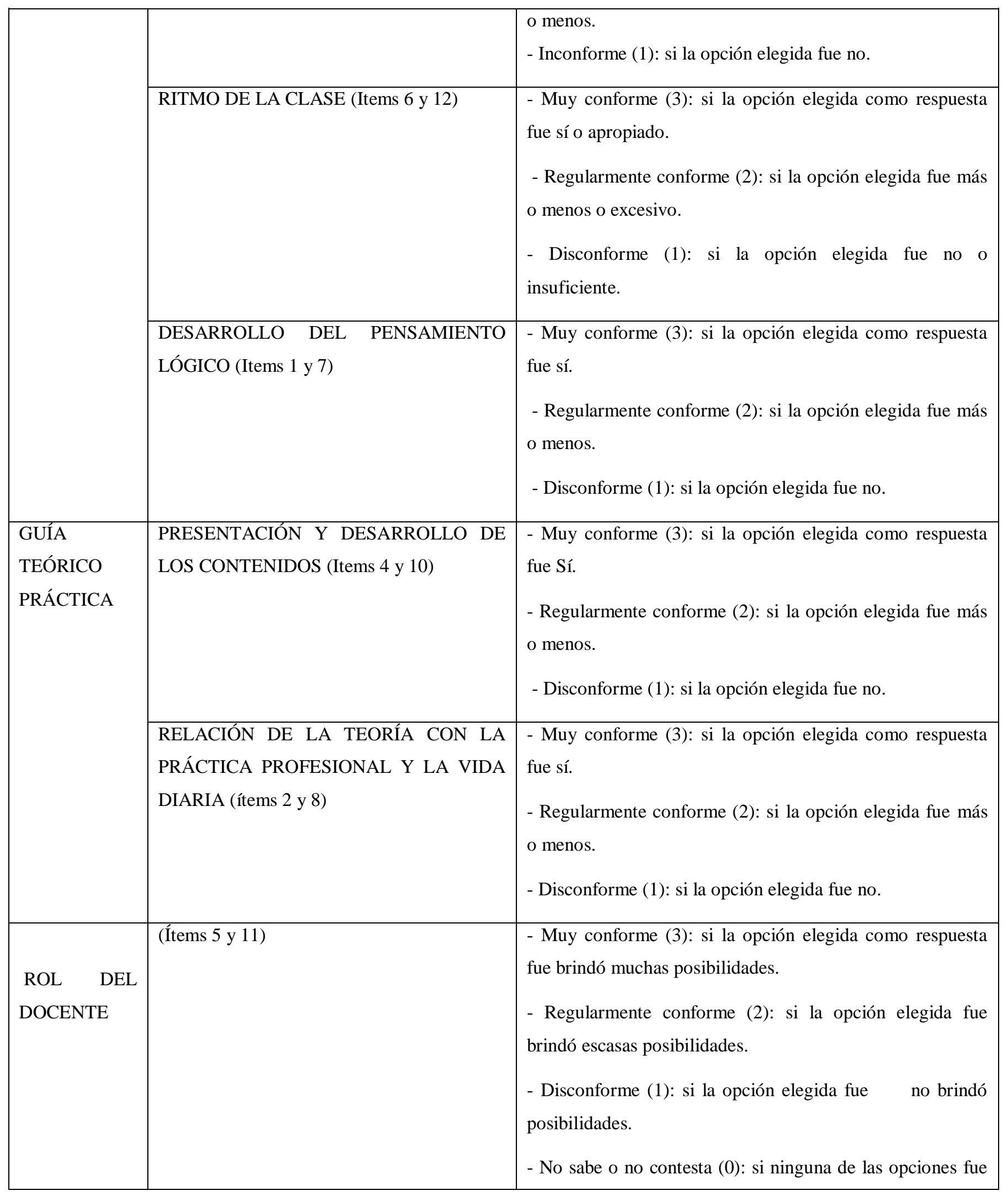


Raquel Vera Velázquez1, Marcos Manuel Manobanda Guamán2, Kirenia Maldonado Zúñiga3, Yoiler Batista Garcet4,Alfredo Lesvel Castro Landin 5

\begin{tabular}{|c|c|c|}
\hline & & elegida \\
\hline
\end{tabular}

\section{ANÀLISIS DE LOS RESULTADOS}

Puesto que la calidad de un estudio y la credibilidad de las conclusiones, están en función de la calidad de los instrumentos que se empleen, se analizó la validez y confiabilidad del cuestionario. Se estudió la validez de contenido del instrumento a través del juicio de expertos. Los cinco jueces seleccionados debían responder a la pregunta “Están contemplados, en los ítems de la encuesta, todos los criterios enunciados para su construcción?” Las opciones de respuestas eran:

1-Adecuadamente contemplado

2- Medianamente contemplado

3-No contemplado

La prueba de rangos de Friedman permitió aceptar la hipótesis nula de concordancia de las valoraciones asignadas por los jueces. E1 90\% de las respuestas correspondió a la categoría: Adecuadamente contemplado. Para favorecer la confiabilidad, el cuestionario que respondido en forma anónima y cada estudiante dispuso del tiempo suficiente para esta tarea. La confiabilidad del cuestionario, considerado en su totalidad, se dedujo de la elevada consistencia interna entre los ítems (de las dos primeras partes) correspondientes a la misma dimensión. Los valores del coeficiente $\alpha$ de Cronbach fueron superiores a 0,85 , salvo uno que dio 0,77 .

En la muestra de 96 encuestados se detectaron, en cuatro de ellos, incoherencias en las respuestas a los dos ítems que medían algunas de las variables. Para cada variable o dimensión, se obtuvieron los porcentajes en cada alternativa establecida como respuesta a los dos ítems formulados para medirla. La última pregunta de la encuesta (única de respuesta abierta) intentaba indagar sobre la afección a la nueva metodología empleada. Al momento de aplicar la encuesta, se solicitó a los estudiantes que en la justificación de esta pregunta hicieran referencia a las distintas dimensiones establecidas: participación y trabajo grupal, ritmo de la clase, rol del docente, desarrollo del pensamiento lógico, presentación y desarrollo de los contenidos y la relación de la teoría con la práctica profesional y la vida diaria. Se obtuvo así información para interpretar los porcentajes obtenidos en las variables y dimensiones para las que se habían formulado preguntas que no requerían justificación. Una vez recopilado los datos, se consideró conveniente conocer la eficiencia de la nueva metodología calculando indicadores, que podrían denominarse "Importancia relativa de cada dimensión". Para ello se obtuvo el valor máximo posible para las dos

104 UNESUM-Ciencias. Publicación cuatrimestral. Vol. 3, Año 2018, No. 2 (agosto) 
preguntas asociadas a una misma dimensión, multiplicando el valor más alto de la escala: 3 (ver Tabla 1) por el número total de protocolos considerados (96), siendo el resultado equivalente al 100\% de este indicador. Luego se calculó el puntaje para las dos preguntas de interés, promediando los valores seleccionados en la escala por todos los encuestados. Finalmente se calculó la importancia relativa de la dimensión en cuestión.

El gráfico 1 presenta los resultados acerca de la eficiencia de la nueva metodología, basándose en la opinión de los estudiantes.

Importancia relativa en \%, de las dimensiones, según opiniones de los alumnos.

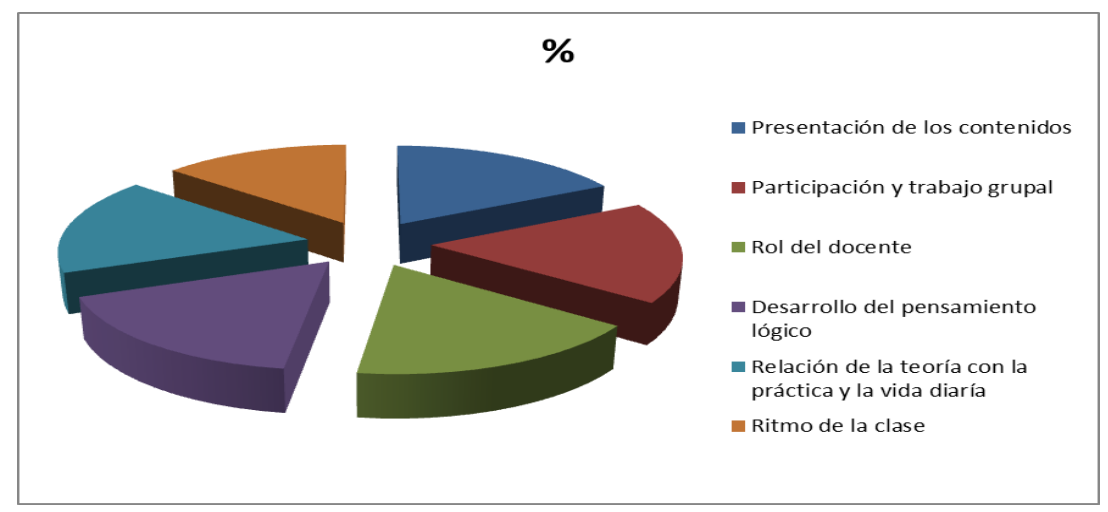

Se puede apreciar que la importancia relativa de las distintas dimensiones osciló entre $65 \%$ y $83 \%$. Los valores más altos estarían indicando, en orden decreciente, que los estudiantes: aprueban el material didáctico propuesto, se muestran complacidos por tener una participación activa en la clase y por el trabajo grupal, están de acuerdo con el rol desempeñado por el docente como guía del aprendizaje y como trasmisor del saber. En una medida algo menor consideran que la nueva metodología favorece el desarrollo del pensamiento lógico y valoran que se haya puesto de manifiesto en la guía la relación existente entre la teoría desarrollada con la práctica profesional y con la vida diaria. Por su parte, el aspecto más débil de esta estrategia didáctica, está relacionado con el ritmo de las clases

Análisis porcentual de las respuestas a las preguntas correspondientes a cada dimensión

Los gráficos muestran las opiniones de los estudiantes, en porcentajes, sobre las distintas dimensiones consideradas. Para cada dimensión se presentan, algunas de las citas textuales más significativas vertidas en la última pregunta del cuestionario.

PARTICIPACIÓN DEL TRABAJO GRUPAL 


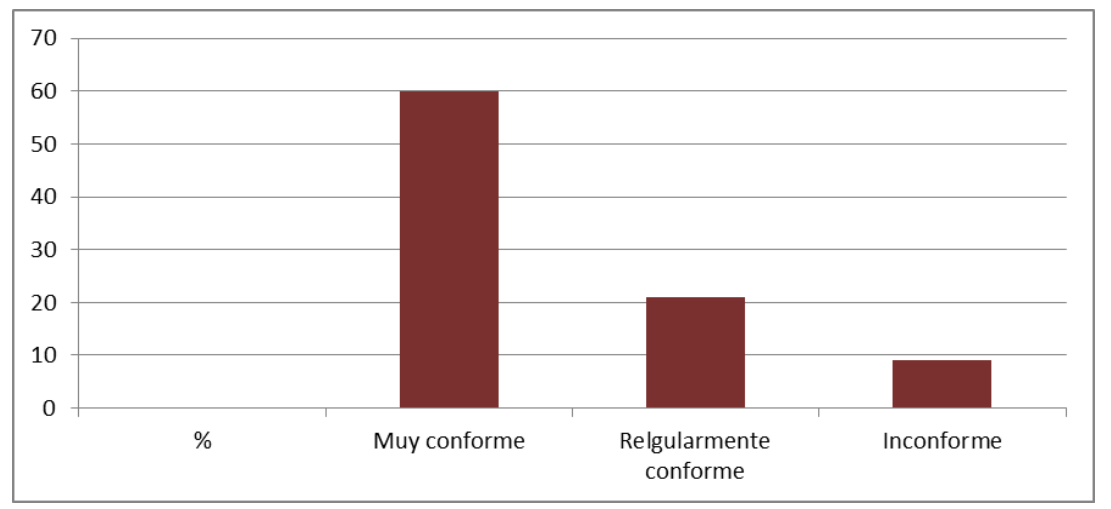

Las respuestas regularmente conformes (23\%) sostenían que les había resultado poco práctico trabajar en grupos. Algunos preferían, por una cuestión de rapidez, que la profesora brindara toda la información. El 7\% de las respuestas inconformes manifestaban que no hubo participación. El $60 \%$ de las opiniones consideradas muy conformes sostenían que en las clases el grado de participación fue alto, y que interviniendo y escuchando a sus compañeros los estudiantes se beneficiaron mutuamente, se facilitó la comprensión de los conceptos, la corrección de los errores y la vía para superarlos, permitiendo que los razonamientos se realizaran en un nivel accesible a todos. Algunos opinaron que el trabajo compartido los desinhibió y logró incrementar su autoestima. Algunas de las opiniones expresadas por los estudiantes fueron: "Sí me gustaría que se desarrollen los próximos temas como ecuaciones y funciones, porque me ayudó a participar y a aprender con el aporte de mis compañeros y del profesor. Además las clases son más fácil y no tan pesadas"; "Sí porque sobre todo el trabajar con mis compañeros y el ver cómo resuelven ellos los problemas y ejercicios me ayudó a entender el tema. La ayuda de la docente y la corrección de los errores también fue importante."; "Cuando un tema es bien desarrollado me ayuda a comprender y razonar cuando tengo que realizar la práctica. Es importante que el profesor dé la posibilidad de participar, porque así aprendo de mis errores y elimino dudas que, a veces, dificultan mi proceder al hacer la práctica. Personalmente valoro una buena teoría y una guía completa y comprensible porque así la práctica es mucho más fácil”

\section{PRESENTACIÓN Y DESARROLLO DE LOS CONTENIDOS}

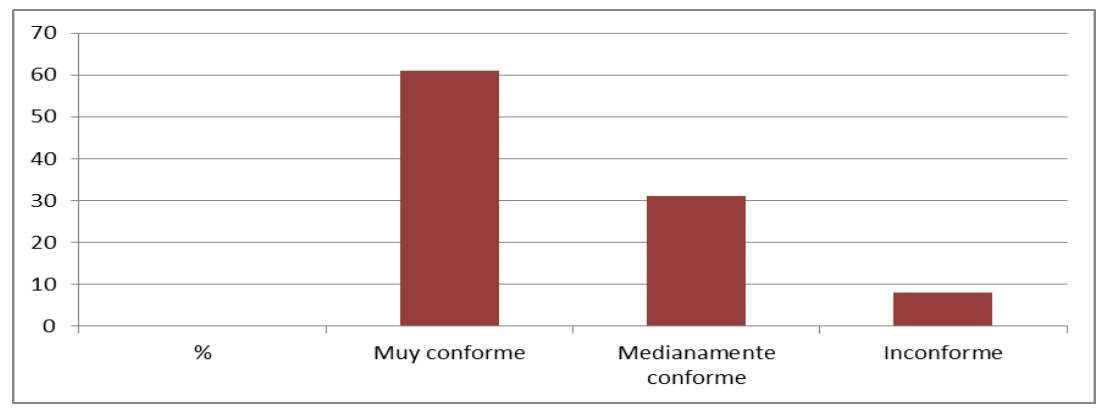

El 31\% Regularmente conforme señaló la necesidad de incluir un mayor número de ejercicios y disponer de más espacio para resolver los problemas. El 62\% Muy conforme sostuvo que este material permitió analizar los temas con todo detalle, tornó necesaria la integración de los conceptos, facilitó la participación y el trabajo con el docente. Destacaron el desarrollo 
fundamentado de los contenidos y la integración de la teoría con la práctica. Algunas de las opiniones fueron: "Cuando un tema es bien desarrollado me ayuda a comprender y razonar cuando tengo que realizar la práctica", "Personalmente valoro una buena teoría y una guía completa y comprensible porque así la práctica es mucho más fácil” y solo el 7\% inconforme.

\section{DESARROLLO DEL PENSAMIENTO LÓGICO}

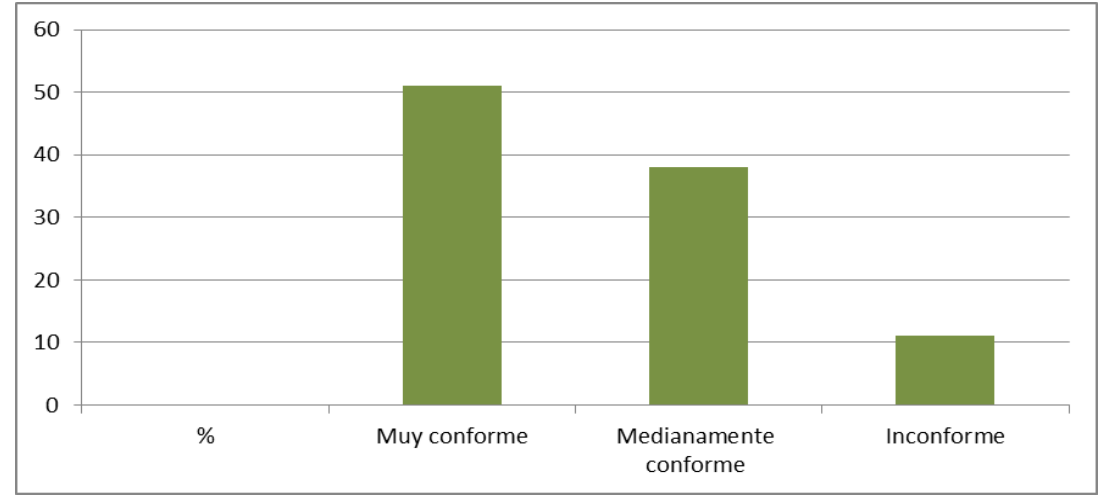

El 38\% regularmente conforme consideró que las clases sólo posibilitaron una pequeña mejora en el razonamiento. El 52\% muy conforme, sostuvo que la guía y la forma en la que se desarrollaron las clases, permitieron realizar distintos tipos de razonamientos y establecer relaciones entre los conceptos. Algunas de las opiniones vertidas fueron: "Me gustó trabajar de esta forma porque me permite extraer mis propias conclusiones y de esa manera razonar más", "En particular siempre me costó aprender matemática, pero creo que ahora estoy aprendiendo a razonar más y a hallar más facilidad para relacionar distintos temas y para resolver problemas", "Creo que en estas clases aprendí mucho gracias a las situaciones propuestas, especialmente en el razonamiento de problemas. El profesor siempre pedía que justifiquemos lo que íbamos afirmando. Por todo esto me gustaría que siempre fuera así”, y el $10 \%$ inconforme.

\section{RELACIÒN DE LA TEORÌA CON LA PRÀCTICA}

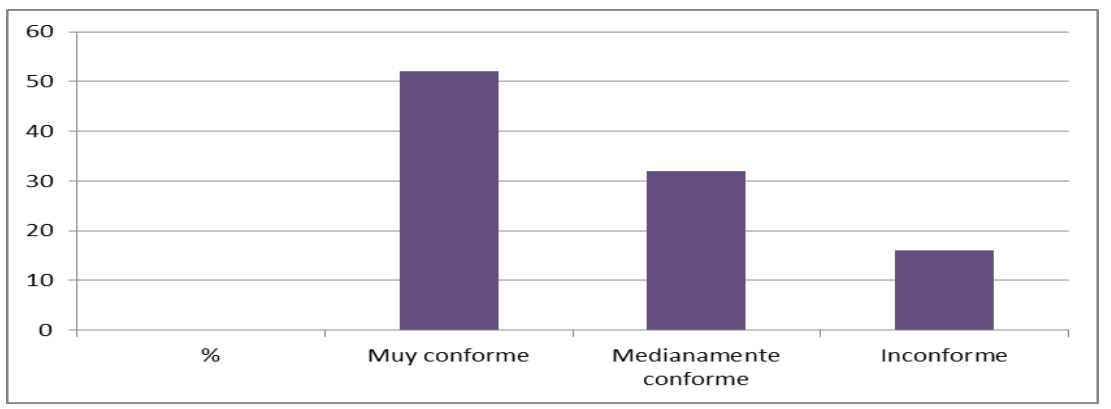

Relación de la teoría con la práctica, presentación y desarrollo de los contenidos: El 33\% regularmente conforme señaló la necesidad de incluir un mayor número de ejercicios y disponer de más espacio para resolver los problemas. $\quad$ El 53\% muy conforme sostuvo que este material permitió analizar los temas con todo detalle, consideró necesaria la integración de los conceptos, facilitó la participación y el trabajo con el docente. Destacaron el desarrollo fundamentado los contenidos y la integración de la teoría con la práctica. Algunas de las opiniones fueron: "Cuando un tema es bien desarrollado me ayuda a 
Raquel Vera Velázquez1, Marcos Manuel Manobanda Guamán2, Kirenia Maldonado Zúñiga3, Yoiler Batista Garcet4,Alfredo Lesvel Castro Landin 5

comprender y razonar cuando tengo que realizar la práctica", "Personalmente valoro una buena teoría y una guía completa y comprensible porque así la práctica es mucho más fácil” y 14\% manifestó que no veía claramente la relación, manteniéndose inconformes.

RITMO DE LA CLASE

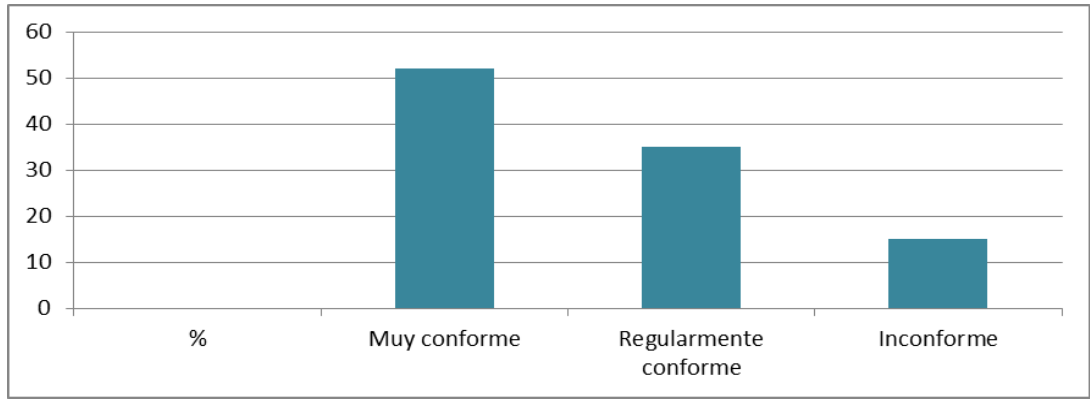

El 33\% de las respuestas catalogadas en regularmente conforme, expresaron: "Me pareció que el tiempo era muy corto y a pesar de que las clases eran entendibles, no bastó para entender a fondo el tema y sacar todas las dudas", "Creo que la metodología con la que se desarrolló el tema está muy buena. Sólo creo que sería más conveniente un poco más de tiempo para su desarrollo". El 15\% inconforme expresó: “El tiempo fue poco y el ritmo muy rápido”, "El tiempo que se empleó para cada punto de continuidad no fue el adecuado (fue poco y rápido). Es un tema lindo, debería ser más lento para poder captar los contenidos". Sin embargo, el 52\% de los estudiantes estuvieron muy conformes, expresando: "Me gustaría que me enseñen todos los temas como lo hicieron con ecuaciones porque se dispuso del tiempo necesario para cada tema “,”En todos los temas tendrían que dar el tiempo necesario como lo hicieron en este".

\section{ROL DEL DOCENTE}

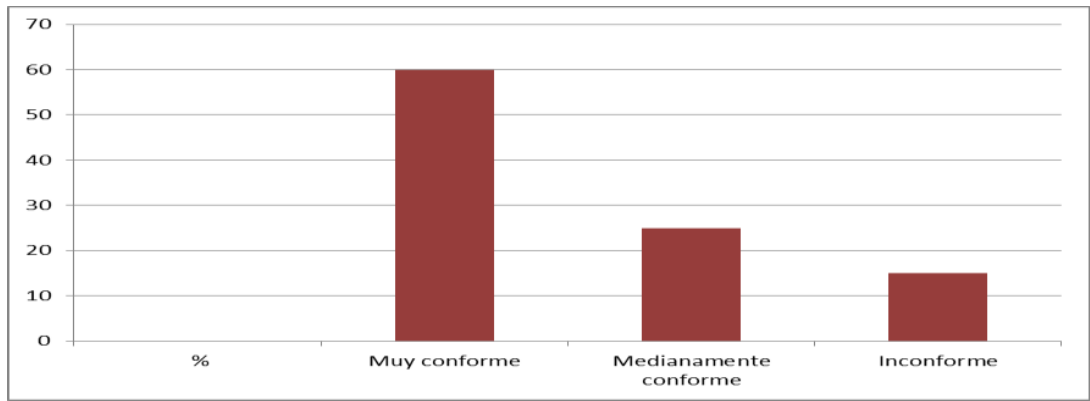

El 25\% consideró medianamente el rol del docente como facilitador, el $60 \%$ estuvo muy conforme, consideró que el docente cumplió el rol de facilitador del proceso de aprendizaje, logrando motivar a los estudiantes y crear un clima distendido en el aula. Encontraron positiva la disminución de su protagonismo al haber concedido importancia a las opiniones de los estudiantes. Vertieron expresiones tales como: "Me pareció importante y necesario el apoyo de la profesora, la forma en que explica y corrige los errores. Y además, el trabajo con los compañeros me ayuda a entender mejor", "Sí, me gustaría volver a

108 UNESUM-Ciencias. Publicación cuatrimestral. Vol. 3, Año 2018, No. 2 (agosto) 
trabajar así porque me resulta mucho más eficiente que el profesor trabaje junto a los estudiantes en las clases y finalmente resalte lo que considere importante que sepamos"y el 15\% se mantuvo inconforme con el rol del docente.

\section{NUEVA METODOLOGÍA}

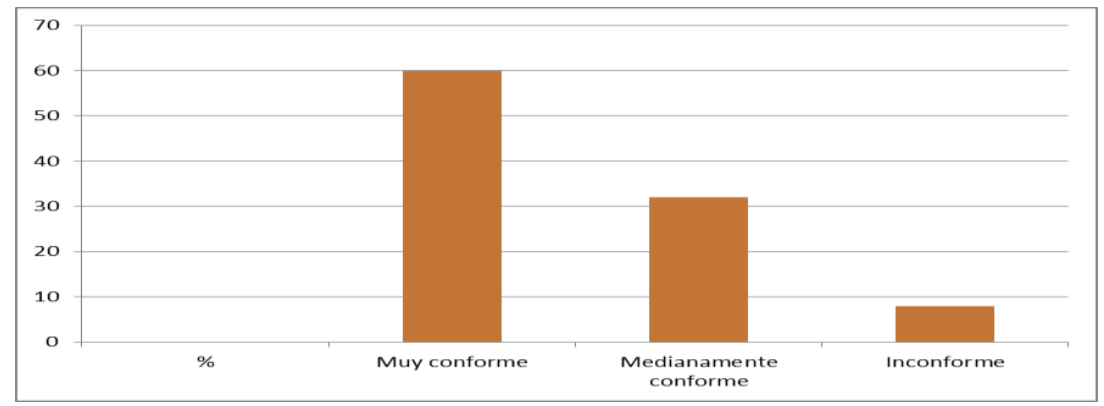

El 60\% manifestó una afección plena, considerando que se favoreció la comprensión y solidez de los conocimientos, la participación activa de los estudiantes al estimularlo a completar la guía, se logró despertar el interés y la atención, se favoreció un aprendizaje constructivista al inducirlos a descubrir los conceptos, definiciones y teoremas. Señalan como positivo el enfoque integrador. Algunas de las opiniones fueron "Sí, porque es una forma diferente de aprender y es más interactivo, lo que hace menos aburrido una clase teórica", "Si, me gustaría que los otros temas se den así puesto que de esta forma la teoría no es tan abstracta“. El 8\% inconforme con la nueva metodología tuvieron expresiones como la siguiente: "Me gustaría que las clases fueran más dinámicas”y el 32\% medianamente conforme.

\section{DISCUSIÒN}

Los resultados obtenidos nos permiten comprender cambios sustantivos en las prácticas pedagógicas en el aula. El porcentaje superior al 52\% ubicado en el grado muy conforme (en casi todas las variables y dimensiones), permitiría concluir que la mayoría de los estudiantes estarían satisfechos con la nueva metodología, e incitaría a aplicarla en la totalidad de los contenidos que integran el currículo de la asignatura. Dado el alto grado de conformidad de los estudiantes en lo relativo a "Participación y trabajo grupal" se aprecia una modificación importante en el tiempo de clase dedicado a las distintas actividades. Este cambio es consecuencia de la estructuración de la estrategia didáctica, que destina gran parte de la sesión al trabajo de los estudiantes, quedando reducida la exposición oral del profesor sobre el contenido matemático que los estudiantes debían aprender (Institucionalización del saber), instancia en la cual el docente tiende puentes entre los conocimientos consensuados en la discusión plenaria y los saberes institucionales que son objeto de enseñanza. De esta manera la estrategia posibilitó, que el docente desempeñara un rol diferente, brindando apoyo al estudiante y estableciendo interacciones con él para señalar errores, promover conexiones entre los distintos contenidos, favoreciendo de esta manera aprendizajes significativos. Estas conclusiones se avienen con lo que sostienen Gil (1987) y Campanario y Moya (1999), al interpretar que el docente es quien debe supervisar el trabajo de los grupos, ofrecer ayudas puntuales cuando sea necesario, estar atento al desarrollo de las actividades y coordinar la puesta en común, reformulando los resultados encontrados por los estudiantes, institucionalizando así los saberes que son objeto de enseñanza. Los estudiantes manifestaron que el docente estimuló la argumentación como elemento de validación de sus 
Raquel Vera Velázquez1, Marcos Manuel Manobanda Guamán2, Kirenia Maldonado Zúñiga3, Yoiler Batista Garcet4,Alfredo Lesvel Castro Landin 5

producciones, evidenciándose preocupación por el desarrollo del pensamiento lógico formal y la comprensión cabal de las ideas matemáticas. Se pondría de manifiesto el interés del docente porque la validez del conocimiento matemático tratado en clase, recaiga en la racionalidad de la Matemática. Puede interpretarse que hubo avances en la motivación y disposición de los estudiantes hacia el aprendizaje esta disciplina, ya que más de la mitad de los encuestados manifestaron satisfacción al haberse involucrado en la resolución de problemas con planteamientos de contextos de la vida real y de la futura práctica profesional. Se confirmarían así las reflexiones sobre la motivación, basadas en las teorías cognitivas, que sostienen que uno de los rasgos que define el interés intrínseco por una tarea o un contenido, es la aplicabilidad percibida del mismo y su utilidad para resolver problemas o situaciones de interés. Aún cuando Afonso López et al (1998) afirman que los estudiantes consideran a los docentes de matemática como los responsables de generar el clima menos adecuado para el trabajo en el aula, pareciera que la nueva estrategia descalificaría esta aseveración. Atendiendo a las críticas manifestadas por los estudiantes en relación al ritmo rápido de las clases, es necesario reconocer que la excesiva cantidad de contenidos a desarrollar en la asignatura determinaron que el tiempo destinado a cada uno fuera muy escaso. Estas críticas estarían de acuerdo con las opiniones de Campanario y Moya (1999) cuando afirman que los enfoques alternativos a la enseñanza tradicional requieren un tiempo más prolongado para desarrollar los contenidos, recomendando reducir los programas de las asignaturas. También, el elevado número de alumnos dificultó un intercambio próximo al diálogo, entre el docente y los alumnos y entre los alumnos entre sí, aun cuando éstos disponían de mayor tiempo para trabajar en forma individual y grupal, y la estrategia se había diseñado desde una visión del aprendizaje centrada en la interacción social. Se aprecia satisfacción con el material instruccional elaborado, habiéndose limitado las observaciones de los alumnos a la necesidad de incorporar un mayor número de problemas y no a cuestiones de incomprensión del contenido por la terminología empleada, por el nivel de complejidad de las actividades ni por la manera en que se abordó el contenido matemático. Con el uso del material instruccional, se coincidiría con Sánchez y Valcárcel (1993), cuando sugieren para la selección de estrategias didácticas, el diseño de una secuencia global de enseñanza y la elaboración de materiales de aprendizaje. Por lo expuesto se aprecia un avance en la práctica docente usual en esta asignatura, tornándose necesario tomar decisiones con respecto a los tópicos matemáticos que se consideran relevantes que el alumno aprenda, a la formación matemática (contenidos conceptuales, procedimentales y actitudinales) que se quiera lograr en el estudiante, de tal manera que la labor del docente de enseñar no tenga una sobrecarga cognitiva innecesaria. Es de destacar que los estudiantes, sin conocer los fundamentos teóricos de la propuesta didáctica, comparten en un alto grado los criterios establecidos para la enseñanza de la matemática.

\section{CONCLUSIONES}

Podemos concluir que se cumplieron, en gran medida, los criterios derivados del marco teórico, los que actuaron como objetivos a lograr durante el desarrollo del proceso de enseñanza y aprendizaje. Ya que según Brousseau (1993) no existe ningún método que por sí solo permita generar en clase un proceso satisfactorio de aprendizaje, es necesario perseverar en la búsqueda de mejores guías para la acción, siempre dispuestos a articular distintas estrategias y asumir las variantes en la relación didáctica que se revelen como necesarias. La búsqueda debe estar dirigida a nuevas estrategias de

110 UNESUM-Ciencias. Publicación cuatrimestral. Vol. 3, Año 2018, No. 2 (agosto) 
trabajo en el aula que animen a los alumnos a ser creativos y de esta manera influir positivamente en sus actitudes. Las mismas, a su vez, pueden tener influencias positivas sobre los resultados del aprendizaje.

La triangulación de las conclusiones de los instrumentos destinados a indagar sobre la práctica didáctica en el aula (guía de práctica y registro del docente) permite afirmar que no existen diferencias en la descripción y valoración de la realidad, realizada a través de ellos. Los altos porcentajes de opiniones muy conforme acerca de las distintas dimensiones consideradas, emitidas por los estudiantes o por el docente, informarían que distintos aspectos de la estrategia didáctica implementada, considerados vitales para favorecer un aprendizaje significativo, han experimentado modificaciones positivas.

\section{REFERENCIAS BIBLIOGRÁFICAS}

Brousseau, G. (1993) Fundamentos y métodos de la Didáctica de la Matemática. Serie B. Trabajos de matemática No 19. Versión castellana. Facultad de Matemática, Astronomía y Física. Universidad Nacional de Córdoba

Campanario, J. M. y Moya A. (1999) “¿Cómo enseñar Ciencias? Principales tendencias y propuestas”. Enseñanza de las Ciencias, 17 (2) pp. 179-192.

Coll, C. y Collomina, R. (1992). Interacción entre alumnos y aprendizaje escolar. En Coll, C., Palacios, J. y Marchesi, A. Desarrollo psicológico y educación, II. Psicología de la educación, pp. 335-354. Madrid: Alianza Editorial.

Gil, D. (1987) Los programas-guía de actividades: Una concreción del modelo constructivista de aprendizaje de las ciencias. Investigación en la Escuela, 3, pp. 3-12.

González de Galindo, S. (2003) Resignificación de las clases teóricas, en una Facultad de ciencias, dentro de un nuevo modelo de aprendizaje. Tesis de Magíster no publicada. Universidad Nacional de Tucumàn Argentina.

González de Galindo, S. y Villalonga de García, P. (2002) Análisis crítico de un currículo de Matemática. Educación y Ciencia, 39-47. Vol. $6 \mathrm{~N}^{\circ} 11$ (25).

González de Galindo, S. y Villalonga de García, P. (2007) Evaluación de la práctica docente de un curso universitario mediante el diario del profesor. Unión, Revista Iberoamericana de Educación Matemática, No 9, pp. 203-221.

González de Galindo, S.; Marcilla, M. y Villalonga de García, P. (2006) Diseño de una Estrategia Didáctica para cursos multitudinarios del nivel superior. Resúmenes de la VI Conferencia Argentina de Educación Matemática SOAREM - pp $11-12$.

González Pérez, M. (2000) Evaluación del aprendizaje en la enseñanza universitaria. Cuba: CEPES, Universidad de la Habana.

González, S.; Villalonga, P.; Marcilla, M. y Mercau, S. (2006) Metodologías de Enseñanza y Evaluación que favorecen aprendizajes significativos para cursos multitudinarios de primer año de una Facultad de Ciencias. VIII Simposio de Educación Matemática. Volumen CD., pp 1555-1570.

Gregg, J. (1995) The tensions and contradictions of de school mathematics trasdition. Journal for Research in Mathematics Education, 26 (5), 442-466.

Hernández Fernández, H. (2001) "Vigotsky y la estructuración del conocimiento matemático. Experiencia cubana". En Hernández Fernández, H., Delgado Rubí, J., Fernández de Alaíza, B., Valverde Ramírez, L. y Rodríguez Hung, T. Cuestiones de didáctica de la matemática. Conceptos y procedimientos en la Educación Polimodal y Superior, pp. 33-54. Argentina: Homo Sapiens Ediciones.

Marcilla, Mercau, Gonzalez y Villalonga (2005) "Opiniones de alumnos universitarios de primer año sobre las clases masivas de matemática". Actas de las I Jornadas de Ciencia y Tecnología de las Facultades de Ingeniería del NOA. Volumen CD.

Moscoso Canabal, J. A. (2005) En torno a la institucionalización del saber matemático en el aula: el caso de la reforma curricular mexicana de 1993. Revista Iberoamericana de Educación Matemática, No 4, pp. 5-16. 
Raquel Vera Velázquez1, Marcos Manuel Manobanda Guamán2, Kirenia Maldonado Zúñiga3, Yoiler Batista Garcet4,Alfredo Lesvel Castro Landin 5

Ortiz Hurtado, M. (1999) Iniciación de la aritmética. Una propuesta de formación de maestros desde la perspectiva del aprendizaje. Tesis de Doctor en Ciencias con especialidad en Matemática Educativa. Centro De Investigaciones y de Estudios Avanzados del Instituto Politécnico Nacional. Departamento de Matemática Educativa. México, D.F.

Pérez Gómez, A. (1992) Los procesos de enseñanza - aprendizaje: análisis didáctico de las principales teorías del aprendizaje. En Gimeno Sacristán y Pérez Gómez (Eds), Comprender y transformar la enseñanza.Madrid: Editorial Morata

Samaja, J. (2003) Epistemología y metodología. Elementos para una teoría de la investigación científica. Buenos Aires: Eudeba.

Sánchez, G. y Valcárcel, M. V. (1993). Citado por Campanario, J. M. y Moya A. “¿Cómo enseñar Ciencias? Principales tendencias y propuestas”. Enseñanza de las Ciencias, 17 (2) pp. 179-192. 1999.

Villalonga de García, P. (2003) Un enfoque alternativo para la evaluación del Cálculo en una Facultad de Ciencias (Tesis de Magíster no publicada. Universidad Nacional de Tucumán. Tucumán). Argentina.

Villalonga de García, P. y González de Galindo, S. (2005) Criterios derivados de teorías cognitivas, empleados como referentes al diseñar y validar una experiencia didáctica. Ponencia de la V Conferencia Argentina de Educación Matemática. Buenos Aires, Argentina.

112 UNESUM-Ciencias. Publicación cuatrimestral. Vol. 3, Año 2018, No. 2 (agosto) 